\title{
Long-term Effects of a Group Community- based Constraint-induced Movement Therapy on Motor Recovery and Activities of Daily Living in Community-dwelling Older Adults with Chronic Stroke: A Preliminary Study with 6-month Follow-Up
}

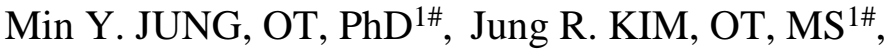 \\ Eun Y. YOO, OT, $\mathrm{PhD}^{1}$, Hye S. JEON, PT, $\mathrm{PhD}^{2}$, \\ Joshua H. YOU, PT, $\mathrm{PhD}^{2}$ \\ ${ }^{1}$ Department of Occupational Therapy, Yonsei University \\ ${ }^{2}$ Department of Physical Therapy, Yonsei University
}

\begin{abstract}
The standard one-on-one constraint-induced movement therapy (CIMT) recently gained widespread acceptance as a means of effective stroke rehabilitation, but inherent issues associated with cost-effectiveness, safety, lack of dynamic group interaction, and compliance hampered this novel approach. This preliminary study highlights the effect of the group community-based CIMT, which integrated the concepts of the standard CIMT regimen. Eight participants were recruited from a local city community center and trained in the usage of mittens and safety management on the affected upper extremity for $90 \%$ of the hours when awake. The community-based CIMT was provided for 2 hours a day over 10 days. Outcome measures include motor recovery, strength, amount of use and quality of movement, self-perceived occupational performance and satisfaction as well as other psychological factors such as motivation, compliance, and enjoyment. These results all showed significant improvement after intervention and were maintained even after the 6-month follow up $(P<0.05)$.
\end{abstract}

Key words: Canadian occupational performance measure, constraint-induced movement therapy, motor recovery, stroke

Received: May 15, 2008, Accepted: February 13, 2009

Correspondending to: Joshua (Sung) H. You, PT, PhD, Associate Professor, Department of Physical Therapy, Graduate School of Rehabilitation Science, Yonsei University, Director of Virtual Reality Education \& Research Center/Center for Movement Impairment Solutions, 234 Heoungup-Myon, MaeJi-Ri, Wonju City, Kangwon-do, Republic of S. Korea 220-710

Phone: 82-33-760-2476 fax: 82-33-760-2496

e-mail: neurorehab@yonsei.ac.kr

\#These authors (Dr. J and Ms K) equally contributed to this work.
(Asian J Occup Ther 7: 1-11, 2009)

\section{Introduction}

Grasping and manipulation are an important basis for normal fine motor skills associated with preparing a meal, gardening, and handwriting (Kwakkel, 1999). In stroke, however, these motor functions are commonly affected (Carr et al., 
1998). As many as $70-80 \%$ of all stroke survivors continue to suffer from this residual fine motor dysfunction even after they regained functional independence in ambulation (Wade et al., 1983). Hemiparetic stroke patients tend to compensate with the unaffected or less affected hand rather than re-utilizing the affected hand in their activities of daily living (ADL) (Taub et al., 2006). Subsequently, this leads to disuse or learned nonuse of the affected limbs and further impairs fine motor skills in the involved limb (Taub et al., 2006). To restore lost motor skills, improve quality of life, and reduce the long-term socioeconomic cost from stroke, annual cost for stroke is estimated to be as much as 30-40 billion dollars (Dobkin, 1995). Hence, there is a great demand for cost-effective stroke intervention to improve motor recovery and skills of the affected limbs.

To improve motor recovery, different neurorehabiliation approaches, including neurodevelopmental treatment (NDT) (Dickstein et al., 1986), muscle reeducation training using functional electrical stimulation (Handa et al., 1987) biofeedback training (Wolf et al., 1983), virtual reality, strength training (Ada et al., 2006), and a various array of constraint-induced movement therapy (CIMT) have been used, but the outcome results were variable (Page et al., 2002; Taub et al., 1999). Among them, the CIMT evolved and accumulated sufficient evidence as an effective intervention paradigm to address the learned non-use of the involved hand, associated muscle weakness and motor skills. For example, an intensive, one-on-one CIMT was found to produce measurable functional motor improvement in a child with a hemiparetic cerebral palsy (Gordon et al., 2005) and adults with chronic hemiparesis (Liepert et al., 1998), but its practicality in a clinical setting warrants further study due to cost-effectiveness, safety, and compliance issues (Page, 2002; Underwood et al., 2006). It was suggested that a community-based CIMT program could help reduce socio-economic costs and also increase accessibility for a number of community dwelling people suffering from chronic hemiparetic stroke (Pang et al., 2006).

To overcome these potential limitations with the conventional CIMT (Hakim et al., 2005; Yen et al., 2005), we have developed a group community-based CIMT paradigm where eight hemipareitc stroke patients were grouped and given CIMT for 2 hours a day, 5 times a week over 2 weeks at a local health community center. Our specific aim was to investigate whether the community-based group CIMT was effective in improving motor recovery of the affected upper extremity, muscle strength, and associated occupational performance. Motor recovery, muscle strength, hand dexterity, occupational performance and satisfaction associated with ADL were assessed by the standardized Fugl-Meyer assessment (FMA), strength test, gross motor dexterity, and Canadian occupational performance measure (COPM), respectively at pretest, posttest, and a 6-month follow-up test. Additionally, a post-intervention survey about enjoyment, motivation, meaningfulness, compliance, accessibility, social interaction, and fatigue related with the participation in therapy was implemented.

\section{Methods}

\section{Participants}

A convenience sample of 8 older adults with chronic hemiparetic strokes who met the inclusion criteria for CIMT was recruited from the local community. Initially, the total number of 120 target population who registered in the handicap beneficiary recipient list of a local city health center in the city of Wonju was screened for eligibility via a telephone interview. Of 120 , only 8 subjects who met the inclusion and exclusion criteria provided signed consent before the participation. The inclusion criteria for CIMT encompass: 1) $\geq 6$ months post-stroke onset; 2) the ability to perform $45^{\circ}$ shoulder flexion and abduction, $90^{\circ}$ elbow flexion and $20^{\circ}$ wrist extension, and $10^{\circ}$ finger range of motion (ROM) (Taub et al., 1999). Exclusion criteria included severe cognitive ( $>22$ in the mini-mental state examination, MMSE) and verbal (e.g., receptive aphasia) dysfunctions and non-ambulatory. Additionally, confidentiality was protected to comply with the requirements of ethics and national laws. For example, dummy code procedure and security cabinet were used to 
Table 1. Demographic and clinical characteristics of the participants

\begin{tabular}{|c|c|c|c|c|c|}
\hline Participants & Age & Gender & paretic side & Dominant hand & $\begin{array}{c}\text { Post-stroke onset } \\
\text { time (months) }\end{array}$ \\
\hline 1 & 63 & $\mathrm{~F}$ & $\mathrm{R}$ & $\mathrm{R}$ & 6 \\
\hline 2 & 68 & M & $\mathrm{R}$ & $\mathrm{R}$ & 144 \\
\hline 3 & 68 & M & $\mathrm{R}$ & $\mathrm{R}$ & 48 \\
\hline 4 & 67 & M & $\mathrm{L}$ & $\mathrm{R}$ & 60 \\
\hline 5 & 71 & M & $\mathrm{R}$ & $\mathrm{R}$ & 24 \\
\hline 6 & 38 & M & $\mathrm{L}$ & $\mathrm{R}$ & 33 \\
\hline 7 & 65 & $\mathrm{~F}$ & $\mathrm{~L}$ & $\mathrm{R}$ & 24 \\
\hline 8 & 65 & M & $\mathrm{L}$ & $\mathrm{R}$ & 48 \\
\hline Mean \pm SD & $63.13 \pm 10.44$ & & & & $48.38 \pm 42.23$ \\
\hline
\end{tabular}

SD: standard deviation, F: female, M: male, R: right, L: left.

protect identification of participant and safe security. Subjects' demographic and clinical characteristics are shown in Table 1.

\section{Intervention}

Intervention was provided at a local the community health clinics of Kangwon province, South Korea. As with the conventional CIMT developed by Taub (2000), the concept and principles of our community-based group CIMT consisted of two components. First, the participants were educated in the usage of restraining mittens and safety management on the unaffected upper extremity for $90 \%$ of the hours when awake. Secondly, "shaping” of the CIMT training procedures was then initiated (Morris et al., 2006). Experienced therapists in CIMT provided the intervention for 2 hours a day over 10 days. "Shaping" is a form of motor behavioral technique to develop a target motor behavior by means of presenting motivating and functional activities to the participants (Uswatte et al., 2006). Specifically, this self-directed CIMT exercise included (1) moving objects from one shelf to another; (2) throwing little balls in a bucket; (3) delicate motor practice such as fastening nuts on bolts, putting pegs in a board, buttoning and unbuttoning, rubbing and pressing therapeutic putty; (4) using a spoon and chopstick; (5) brushing of teeth, and so on. Before the intensive training, the participants and the therapist met as a group to discuss efforts how to effectively use the more affected hand, exercised the day before at home, and also to encourage their efforts to continue participating in the training period.

Positive feedback including visual, verbal or tactile cues was given to direct the desirable motor skill acquisition whereas positive reinforcement including immediate and frequent praise or cheering, supportive gestures was provided whenever the participants made meaningful efforts and progressed toward a novel task with an increase in the utilization of the affected extremity (Smania, 2006). Based on the motor learning practice paradigm, functional reaching, grasping, and manipulation tasks along with bearing weight on the arm were broken down in ways that each participant could start practicing on an individual component of the whole task and systematically work toward the entire task (Shumway-Cook et al., 2001). Once the adult successfully acquired a novel motor skill, the therapist challenged him or her to internalize this by gradually increasing the motor task demands for accuracy, fluidity, consistency, strength, coordinated automaticity, and ecological carry-over (Morris et al., 2006). The CIMT was also integrated with daily tasks including preparing a meal, shaving, dressing, eating, and grooming in typical home situations. Shaping motor skills were developed considering (1) client centered goals, motivational and meaningful activities, age- and gender-appropriate self-help skills and independence as determined by the client-centered COPM assessment; and (2) 
therapeutic exercises that therapists recommended for optimal functional improvement (Taub, 2000). No additional treatment was provided because all the participants had already completed a routine course of stroke rehabilitation before their participation of this intervention.

\section{Clinical measurements}

The pre-test, post-test, and follow-up-tests were scheduled at baseline, 2 weeks after the baseline test, and a 6-month post-intervention, respectively. The standardized motor recovery, muscle strength, and functional motor skill tests were used to determine intervention-related changes in motor recovery, strength, and associated daily living activities and motor skills of the affected upper extremity. In addition, we performed a survey about enjoyment, motivation, meaningfulness, and compliance. The standardized tests included the Fugl-Meyer assessment (FMA), hand grip and pinch strength test, box and block test, and the Canadian occupational performance measure (COPM). The validity and reliability for these standardized tests are well established (Desrosiers et al., 1994; FuglMeyer et al., 1975; Law et al., 1990; Mathiowetz et al., 1985). The mean value of three successive trials was calculated in all tests and was used in further statistical analysis.

Fugl-Meyer Motor Assessment (FMA): FMA was used to assess motor recovery of the affected upper extremity and hence only the upper extremity subset of FMA was utilized. The upper extremity subset of FMA includes range of motion, pain, sensation, reflex, synergy, coordination, and speed. The scores ranged from 0 (lowest) to 66 (highest) points (Fugl-Meyer et al., 1975).

Box and Block Test (BBT): The box and block test was used to measure unilateral manual dexterity. The test involves a hand motor performance task that requires moving as many blocks as possible from one container to the other within one minute. An increase in the number of blocks moved represents better performance in manual hand dexterity (Mathiowetz et al., 1985).

Hand strength: Grip and pinch strength were measured with a Jamar dynamometer (Asimow
Engineering, US) and pinch gauge (B \& L Engineering, US), respectively. For both tests, the subjects were asked to sit on a chair with shoulder adduction, $90^{\circ}$ elbow flexion and the wrist in a neutral position. The subjects were then instructed to maximally grip the dynamometer handle for 5 seconds without any compensatory movement. The pinch strength was determined by asking the subjects to maximally press a pinch gauge for 5 seconds (Mathiowetz et al., 1985).

Activity of Daily Living: Canadian Occupational Performance Measure (COPM) : COPM was used to determine a client-centered occupational performance associated with activities of daily living. In the present study, the standardized COPM was used to assess functional carry-over or transfer effects of meaningful occupational performance of each client or individual. Compared to motor activity log (MAL), the advantage of this test is that it involves a semi-structured interview which measures both self-rated occupational performance and satisfaction in the domains of self-care, productivity, and leisure. Therefore, this test reflects the client's actual ADL performance and satisfaction accurately which cannot be measured by MAL. From this test, the top five problem areas were identified and self-rated by each individual based on a scale from 1 (unable to perform) to 10 (able to perform) and for satisfaction from 1 (not satisfied) to 10 (satisfied)(Carpenter et al., 2001; Law et al., 1994).

Post-intervention survey: Upon the successful completion of the intervention, all subjects were instructed to complete a postintervention survey that asked about enjoyment, motivation, meaningfulness, compliance, accessibility, social interaction, and fatigue associated with therapy. The scale ranges from 1 (worst) to 4 (best).

\section{Statistical analysis}

The independent variable was a group community-based CIMT and dependent variables included motor recovery, strength, and occupational performance and satisfaction measures. The mean and standard deviation, and 


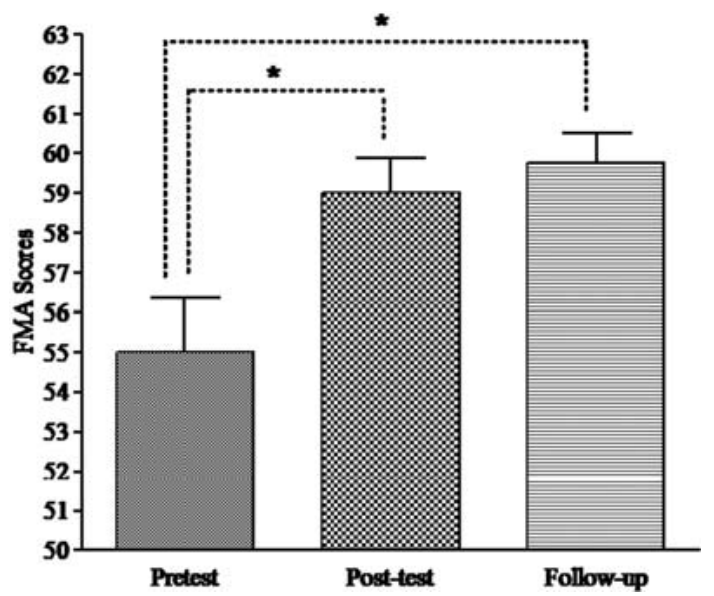

Fig. 1. Effects of the group community-based CIMT on FMA scores. $* P<0.05$.

one-way repeated analysis of variance (ANOVA) were computed to compare intervention-related changes across three tests. Post-intervention survey data were analyzed using descriptive statistics. Statistical analysis was performed using SPSS (version 12). All significance level was set at $P<0.05$.

\section{Results}

\section{Motor recovery}

One-way repeated ANOVA analysis revealed a significant difference across the three points of measurement $(P=0.008)$ (Table 3$)$. Post hoc analysis showed that there were significant differences between the pretest and post-test scores $(P=0.012)$ as well as between the pretest and follow-up test scores $(P=0.004)$. However, there were no significant differences between the post-test and follow-up test scores $(P=0.613)$. These findings suggest that the group communitybased CIMT was effective in the restoration of motor recovery of the affected upper extremity and this effectiveness was retained in the follow-up test (Fig. 1).

\section{Hand manual dexterity}

ANOVA analysis showed no significant difference across the three tests $(P=0.822)$.

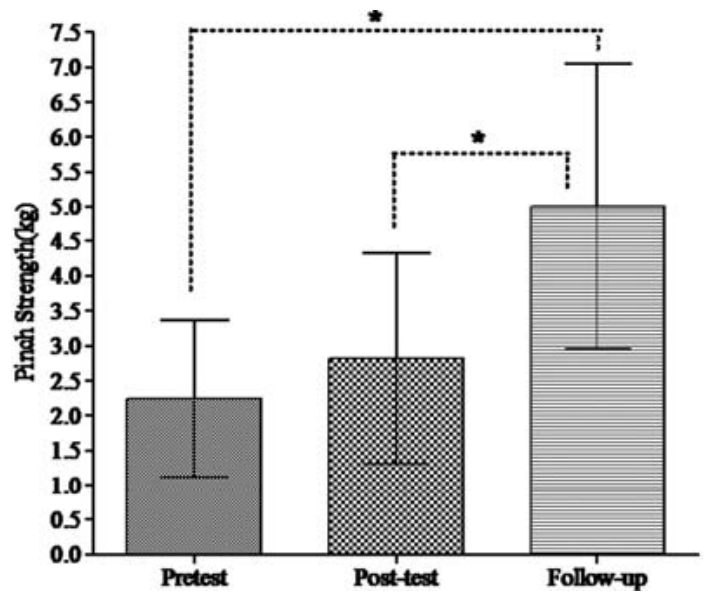

Fig. 2. Effects of the group community-based CIMT on pinch strength. ${ }^{*} P<0.05$.

Specifically, post hoc analysis demonstrated that no significant differences between the pretest and post-test scores $(P=0.580)$ as well as between the pretest and follow-up test scores $(P=0.605)$ were observed. Similarly, these results indicated that the intervention was beneficial in the effective enhancement of manual hand dexterity in individuals with chronic hemiparesis. This intervention effect was maintained even at the 6month follow-up test.

\section{Muscle strength}

Pinch strength data showed significant differences across the three points of measurement $(P=0.006)$ (Table 3). Post hoc analysis confirmed that there were significant differences between the pretest and follow-up test scores $(P=0.002)$ as well as between the post-test and follow-up test scores $(P=0.013)$ (Fig. 2). However, grip strength data analysis was unaffected by this intervention $(P=0.788)$. These results imply that only pinch strength was enhanced as a function of intervention and still remained after postintervention.

\section{Clients' perception of self-rated occupational performance}

Table 4 presents significant intervention effects on the occupational performance and 
Table 2. Individual Fugl-Meyer Motor Assessment data

\begin{tabular}{lccc}
\hline Participants & Pretest & Post-test & 6-month follow-up test \\
\hline 1 & 53 & 59 & 61 \\
2 & 52 & 57 & 57 \\
3 & 60 & 63 & 63 \\
4 & 52 & 56 & 59 \\
5 & 55 & 57 & 57 \\
6 & 58 & 61 & 61 \\
7 & 50 & 58 & 59 \\
8 & 60 & 61 & 61 \\
\hline Mean \pm SD & $55.00 \pm 3.89$ & $59.00 \pm 2.45$ & $59.75 \pm 2.12$ \\
\hline$P$-value & & $0.008^{*}$ & \\
\hline
\end{tabular}

SD: standard deviation, $* P<0.05$.

Table 3. Individual pinch strength $(\mathrm{kg})$ data

\begin{tabular}{lccc}
\hline Participants & Pretest & Post-test & 6-month follow-up test \\
\hline 1 & 0.5 & 0.5 & 3.7 \\
2 & 2 & 2 & 2 \\
3 & 4 & 4.5 & 8.5 \\
4 & 2.7 & 4.5 & 4.5 \\
5 & 2 & 2 & 5 \\
6 & 3.5 & 4 & 5 \\
7 & 1.5 & 1.5 & 4 \\
8 & 1.7 & 3.5 & 7.3 \\
\hline Mean \pm SD & $2.24 \pm 1.13$ & $2.81 \pm 1.51$ & $5.00 \pm 2.05$ \\
\hline$P$-value & & $0.006^{*}$ & \\
\hline
\end{tabular}

SD: standard deviation, $* P<0.05$.

satisfaction scores. A significant difference across the three mean COPM performance scores was found $(P=0.0001)$. Post hoc analysis showed that significant differences between the pretest and post-test scores $(P=0.000)$ as well as between the pretest and follow-up test scores $(P=0.000)$ were observed. However, there was no significant difference between the post-test and follow-up test scores $(P=0.156)$ (Fig. 3). A significant difference across the three mean COPM satisfaction scores was found $(P=0.0001)$. Post hoc analysis showed that significant differences between the pretest and post-test scores $(P=0.000)$ as well as between the pretest and follow-up test scores $(P=0.000)$ were observed. There was a significant difference between the post-test and follow-up test scores $(P=0.021)$ (Fig. 4). These findings suggest that the perceived self-rated occupational performance improved after the intervention and was maintained even in the 6-month follow-up test.

\section{Post-intervention survey}

The survey revealed that all participants enjoyed the social interaction during the program; were highly motivated and supportive each other. They also reported that the therapy was extremely meaningful, realistic, individualized, and functional because it was a client-centered program. All participants showed perfect attendance, indicating an excellent compliance. 
Table 4. Individual Canadian Occupational Performance Measure data

\begin{tabular}{ccccccc}
\hline \multirow{2}{*}{ Participants } & \multicolumn{3}{c}{ Performance scores } & \multicolumn{3}{c}{ Satisfaction scores } \\
\cline { 2 - 7 } & Pre & Post & FU & Pre & Post & FU \\
\hline 1 & 3.70 & 7.70 & 8.60 & 4.30 & 7.30 & 8.60 \\
2 & 6.00 & 6.00 & 7.50 & 3.50 & 4.50 & 7.50 \\
3 & 1.30 & 8.30 & 8.30 & 3.30 & 8.00 & 8.30 \\
4 & 1.70 & 7.30 & 7.30 & 2.30 & 7.30 & 7.60 \\
5 & 2.00 & 7.70 & 7.60 & 3.00 & 8.70 & 8.30 \\
6 & 4.00 & 7.30 & 8.30 & 3.00 & 7.70 & 9.00 \\
7 & 1.80 & 6.30 & 8.00 & 2.00 & 5.30 & 7.80 \\
8 & 2.30 & 4.60 & 6.70 & 2.00 & 4.20 & 7.30 \\
\hline \multirow{2}{*}{ Mean \pm SD } & $2.85 \pm 1.60$ & $6.90 \pm 1.20$ & $7.79 \pm 0.63$ & $2.93 \pm 0.80$ & $6.63 \pm 1.71$ & $8.05 \pm 0.59$ \\
\hline \multirow{2}{*}{ P-value } & \multirow{2}{*}{$0.0001^{*}$} & & $0.0001^{*}$ & \\
\hline
\end{tabular}

SD: standard deviation, Pre: pretest, Post: post-test, FU: 6-month follow-up test, ${ }^{*} P<0.05$.

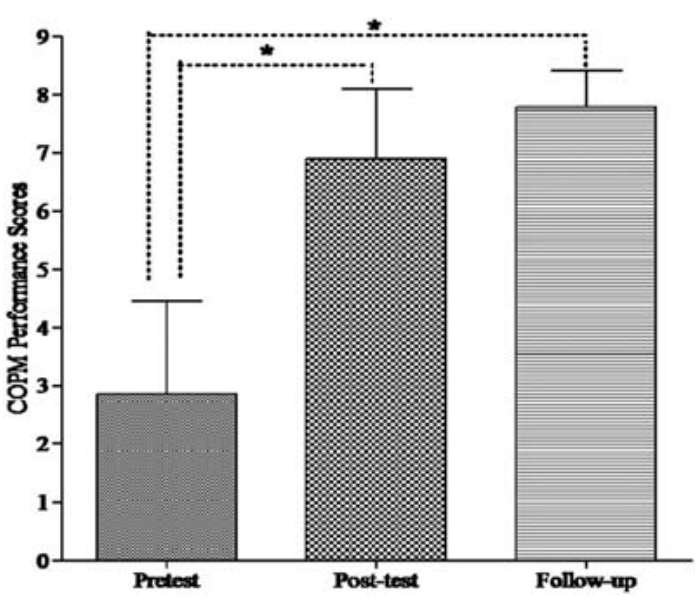

Fig. 3. Effects of the group community-based CIMT on COPM performance. $* P<0.05$.

\section{Discussion}

Our basic assumption was that the group community-based CIMT may improve restoration of motor function, muscle strength, and associated ADL. As anticipated, our results demonstrated that motor recovery and associated ADL skills of the affected upper extremity increased after the intervention and these improvements were

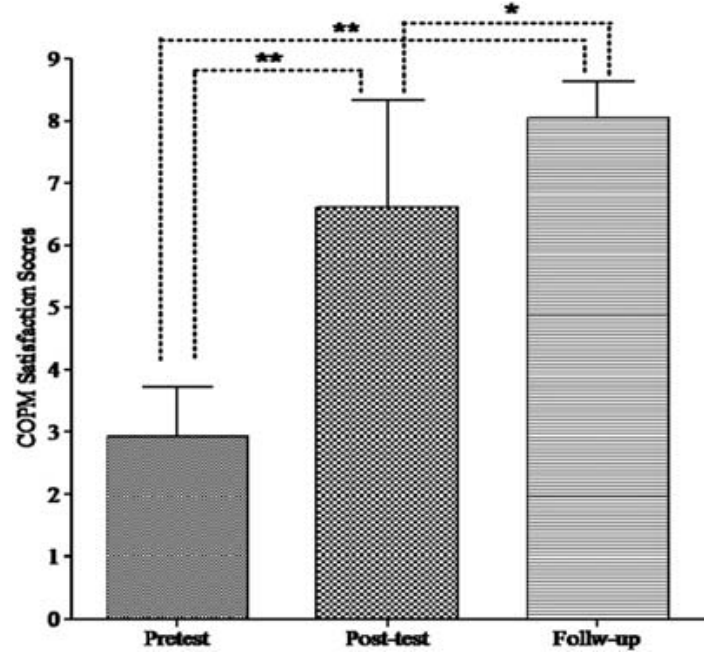

Fig. 4. Effects of the group community-based CIMT on COPM satisfaction. ${ }^{*} P<0.05, * * P<0.001$.

transferred to home environments and retained even during a 6-month follow-up. Certainly, our novel findings suggest that the group communitybased CIMT paradigm can be an alternative approach which can appropriately address the socio-economic and compliance issues raised in the conventional CIMT. The target population is community-dwelling chronic stroke survivors and intervention implemented in community health 
clinics to increase integration into the community.

Despite the fact that our community-based CIMT protocol had much less treatment time (2 hours a day for 2 weeks) and was implemented in a group session, the present study showed promising results which could favorably be compared to previous clinical trials investigating the effects of CIMT in stroke rehabilitation. Several CIMT studies have produced measurable neuroplastic changes and associated functional outcomes in participants with hemiparetic stroke (Bonifer et al., 2005; Dettmers et al., 2005; Sunderland et al., 2005). However, considering the current managed health care model, the CIMT approach which often requires a prolonged individual client-therapist session, and associated cost and compliance issues may not be viable, but rather demands for effective alternative neurorehabilitation programs. For example, it was documented that several undesirable behavioral patterns connected with individual client-therapist session for 5 to 6 hours a day during the standard CIMT resulted in boredom and fatigue coupled with stressful and depressing feelings and emotions (Boylstein et al., 2005). In fact, several patients who participated in the CIMT became depressed and required psychological consultations. On the contrary, in our communitybased group CIMT intervention, we observed that all participants appeared to enjoy the dynamic group interactions which were evidenced by no attrition rate, perfect attendance, good compliance, supportive atmosphere, and positive body gestures (smile and appraise). Perhaps, the group session may have facilitated motivation and encouraged individual participant to obtain his or her goals via pleasant dynamic group interaction (Pang et al., 2006). Additionally, the cost-effectiveness is another advantage of our community-based group CIMT intervention. In fact, we can reduce the current therapy cost by as much as $50 \%$.

In an attempt to offset cost while improving effectiveness that was neither attempted nor afforded by the standard one-on-one CIMT, recent studies (Lum et al., 2004; Page et al., 2005; Pang et al., 2006; Taub et al., 2005) started to modify the conventional CIMT approach. For example, the paradigm shifted from a clinic to a home setting, thus from an individual basis to a small group basis (2-3 persons), which in addition, resulted in reduced supervision time. However, to our knowledge, this is the first attempt examining the effectiveness of a group community-based CIMT on motor recovery, strength and occupational performance and satisfaction. Our FMA results showed significant changes in motor recovery as a function of intervention and this recovery continued to exist even 6 months after initial intervention. This motor recovery was further corroborated by significantly increased pinch strength and hand dexterity function. Similarly, clients' perception of self-rated occupational performance showed that all participants have achieved their established goals and reported higher satisfaction as evidenced by the COPM satisfaction score.

Most importantly, our primary inquiry addressed in this preliminary experiment, was supported by the fact that there were significant differences in the outcome measures of motor behaviors and ADL performance across the three test points. Such evidence seems to imply the notion that both reduced supervision or treatment time and a group-based approach used in our present group community-based CIMT regimen does not preclude a possible advantage or benefit. Further study warrants elucidating the potential equality or superiority in cost-effectiveness between the standard and group community-based CIMTs.

Post-intervention survey data showed that the participants reported psychosoical benefits including enjoyment, motivation, meaningfulness, compliance. For example, we observed no attrition rate, greater interaction with happy smiles, and supporting and encouraging each other. Probably, these benefits may have facilitated engagement on tasks and the amount of use and quality of the movement of the more affected limb during activities, which in turn contributed to motor recovery, strength, and selfperceived occupational performance and satisfaction. 


\section{Limitation of study and suggestions for future study}

This preliminary study explored the feasibility of a community-based group CIMT program for the effective management of upper extremity motor dysfunction and associated grasping and manipulation performances in individuals with chronic stroke. Some shortcomings identified in this preliminary study that could assist in improving the design of future research include small sample size, and lack of a control group. Therefore, precaution should be exercised when interpreting our results. This study invites future research that should be implemented with a robust experimental design and a larger sample size to generalize our findings. It would be of great interest to compare costeffectiveness between the community-based group CIMT and the conventional therapy models in future.

\section{Conclusions}

This present study represents a successful steppingstone toward the development of a group community-based CIMT program. Numerous stroke survivors are financially burdened and often discharged to home without receiving the benefits of an essential long-term rehabilitation program to maximize motor recovery (Lum et al., 2004). Consequently, stroke victims who were not properly rehabilitated may predispose them to fatal falls or other medically debilitating conditions such as pneumonia, contracture, decubitis ulcers, and atrophy due to a lack of physical activity and meaningful occupational performance (Taub et al., 2006). This may lead to a vicious cycle of physical disabilities and socioeconomic constraint to the individual, family, and society at large. The group community-based CIMT program may thus be an alternative choice of intervention that embraces both emerging health promotion and prevention in individuals with chronic disabilities resulting from stroke. In addition, this program can accommodate a large number of community dwelling older adults and does not require individual attention as in the standard CIMT, thereby reducing costs and maximizing therapeutic efficacy.

\section{References}

Ada, L., Dorsch, S., \& Canning, C. G. (2006). Strengthening interventions increase strength and improve activity after stroke: a systematic review. Australian Journal of Physiotherapy. 52, 241248.

Bonifer, N.M., Anderson, K.M., \& Arciniegas, D.B. (2005). Constraint-induced movement therapy after stroke: efficacy for patients with minimal upper-extremity motor ability. Archives of Physical Medicine and Rehabilitation. 86, 18671873.

Boylstein, C., Rittman, M., Gubrium, J., Behrman, A., \& Davis, S. (2005). The social organization in constraint-induced movement therapy. Journal of Rehabilitation Research Development. 42, 263275.

Carr, J., \& Shepherd, R. (1998). Neurological Rehabilitation: Optimizing Motor Performance. Boston: Butterworth-Heineman.

Carpenter, L., Baker, G.A., \& Tyldesley, B. (2001). The use of the Canadian occupational performance measure as an outcome of a pain management program. Canadian Journal of Occupational Therapy. 68, 16-22.

Desrosiers, J., Bravo, G., Hebert, R., Dutil, E., \& Mercier, L. (1994). Validation of the box and block test as a measure of dexterity of elderly people: reliability, validity, and norms studies. Archives of Physical Medicine and Rehabilitation. 75, 751-755.

Dettmers, C., Teske, U., Hamzei, F., Uswatte, G., Taub, E., \& Weiller, C. (2005). Distributed form of constraint-induced movement therapy improves functional outcome and quality of life after stroke. Archives of Physical Medicine and Rehabilitation. 86, 204-209.

Dickstein, R., Hocherman, S., Pillar, T., \& Shaham, R. (1986). Stroke rehabilitation. Three exercise therapy approaches. Physical Therapy. 66, 12331238.

Dobkin, B.H. (1995). The economic impact of stroke. Neurology. 45, S6-S9.

Fugl-Meyer, A.R., Jaasko, L., Leyman, I., Olsson, S., \& Steglind, S. (1975). The post-stroke hemiplegic patient: a method for evaluation of physical performance. Scandinavian Journal of Rehabilitation Medicine. 71, 13-31.

Gordon, A.M., Charles, J., \& Wolf, S.L. (2005). 
Methods of constraint-induced movement therapy for children with hemiplegic cerebral palsy: development of a child-friendly intervention for improving upper-extremity function. Archives of Physical Medicine and Rehabilitation. 86, 837844.

Hakim, R.M., Kelly, S.J., Grant-Beuttler, M., Healy, B., Krempasky, J., \& Moore, S.(2005). Case report: a modified constraint-induced therapy (mCIT) program for the upper extremity of a person with chronic stroke. Physiotherapy Theory Practice. 21, 243-256.

Handa, Y., \& Hoshimiya, N. (1987). Functional electrical stimulation for the control of the upper extremities. Medical Progress Through Technology. 12, 51-63.

Kwakkel, G., Kollen, J.K., \& Wagenaar, R.C. (1999). Therapyimpact of functional recovery in stroke rehabilitation: a critical review of the literature. Physiotherapy. 85, 377-391.

Law, M., Baptiste, S., McColl, M., Opzoomer, A., Polatajko, H., \& Pollock, N. (1990). The Canadian occupational performance measure: an outcome measure for occupational therapy. Canadian Journal of Occupational Therapy. 57, 82-87.

Law, M., Polatajko, H., Pollock, N., McColl, M.A., Carswell, A., \& Baptiste, S. (1994). Pilot testing of the Canadian occupational performance measure: clinical and measurement issues. Canadian Journal of Occupational Therapy. 61, 191-197.

Liepert, J., Miltner, W.H., Bauder, H., Sommer, M., Dettmers, C., \& Taub, E. (1998). Motor cortex plasticity during constraint-induced movement therapy in stroke patients. Neuroscience Letter. 26, 5-8.

Lum, P.S., Taub, E., Schwandt, D., Postman, M., Hardin, P., \& Uswatte, G. (2004). Automated constraint-induced therapy extension (AutoCITE) for movement deficits after stroke. Journal of Rehabilitation Research Development. 41, 249258.

Sunderland, A., \& Tuke, A. (2005). Neuroplasticity, learning and recovery after stroke: a critical evaluation of constraint-induced therapy. Neuropsychology Rehabilitation. 15, 81-96.

Mathiowetz, V., Kashman, N., Volland, G., Weber, K., Dowe, M., \& Rogers, S. (1985). Grip and pinch strength: normative data for adults. Archives of Physical Medicine and Rehabilitation. 66, 6974.

Mathiowetz, V., Volland, G., Kashman, N., \&
Weber, K. (1985). Adult norms for the box and block test of manual dexterity. American Journal of Occupational Therapy. 39, 386-391.

Morris, D.M., Taub, E., \& Mark, V.W. (2006). Constraint-induced movement therapy: characterizing the intervention protocol. Eura Medicophysica. 42, 257-268.

Page, S.J., Levine, P., Sisto, S., Bond, Q., \& Johnston, M.V. (2002). Stroke patients' and therapists' opinions of constraint-induced movement therapy. Clinical Rehabilitation. 16, 55-60.

Page, S.J., Levine, P., \& Leonard, A.C. (2005). Modified constraint-induced therapy in acute stroke: a randomized controlled pilot study. Neurorehabilitation Neural Repair. 19, 27-32.

Pang, M.Y., Harris, J.E., \& Eng, J.J. (2006). A community-based upper-extremity group exercise program improve s motor function and performance of functional activities in chronic stroke: a randomized controlled trial. Archives of Physical Medicine and Rehabilitation. 87, 1-9.

Shumway-Cook, A., \& Woollacott, M.H. (2001). Motor Control: Therapy and Practical Applications. Philadelphia: Lippincott.

Smania, N. (2006). Constraint-induced movement therapy: an original concept in rehabilitation. Eura Medicophysica. 42, 239-240.

Taub, E. (2000). Constraint-induced movement therapy and massed practice. Stroke. 31, 986-988.

Taub, E., Lum, P.S., Hardin, P., Mark, V.W., \& Uswatte, G. (2005). AutoCITE: automated delivery of CI therapy with reduced effort by therapists. Stroke. 36, 1301-1304.

Taub, E., Uswatte, G., Mark, V.W., Morris, \& D.M. (2006). The learned nonuse phenomenon: implications for rehabilitation. Eura Medicophysica. 42, 241-256.

Taub, E., Uswatte, G., \& Pidikiti, R. (1999). Constraint-Induced Movement Therapy: a new family of techniques with broad application to physical rehabilitation-a clinical review. Journal of Rehabilitation Research Development. 36, 237251.

Underwood, J., Clark, P.C., Blanton, S., Aycock, D.M., \& Wolf, S.L. (2006). Pain, fatigue, and intensity of practice in people with stroke who are receiving constraint-induced movement therapy. Physical Therapy. 86, 1241-1250.

Uswatte, G., Taub, E., Morris, D., Barman, J., \& Crago, J. (2006). Contribution of the shaping and restraint components of constraint-induced 
movement therapy to treatment outcome. NeuroRehabilitation 21, 147-156.

Wade, D.T., Langton-Hewer, R., Wood, V.A., Skilbeck, C.E., \& Ismail, H.M. (1983). The hemiplegic arm after stroke: measurement and recovery. Journal of Neurology, Neurosurgery, and Psychiatry. 46, 521-524.

Wolf, S.L., \& Binder-MacLeod, S.A. (1983). Electromyographic biofeedback applications to the hemiplegic patient: changes in upper extremity neuromuscular and functional status. Physical Therapy. 63, 1393-1403.

Yen, J.G., Wang, R.Y., Chen, H.H., \& Hong, C.T. (2005). Effectiveness of modified constraintinduced movement therapy on upper limb function in stroke subjects. Acta Neurology Taiwan. 14, 16-20. 